\title{
Sequence Lorentz Spaces and Their Geometric Structure
}

\author{
Maciej Ciesielski ${ }^{1} \cdot$ Grzegorz Lewicki $^{2}$ \\ Received: 16 May 2018 / Published online: 1 August 2018 \\ (c) The Author(s) 2018
}

\begin{abstract}
This article is dedicated to geometric structure of the Lorentz and Marcinkiewicz spaces in case of the pure atomic measure. We study complete criteria for order continuity, the Fatou property, strict monotonicity, and strict convexity in the sequence Lorentz spaces $\gamma_{p, w}$. Next, we present a full characterization of extreme points of the unit ball in the sequence Lorentz space $\gamma_{1, w}$. We also establish a complete description up to isometry of the dual and predual spaces of the sequence Lorentz spaces $\gamma_{1, w}$ written in terms of the Marcinkiewicz spaces. Finally, we show a fundamental application of geometric structure of $\gamma_{1, w}$ to one-complemented subspaces of $\gamma_{1, w}$.
\end{abstract}

Keywords Lorentz and Marcinkiewicz spaces $\cdot$ Strict monotonicity $\cdot$ Strict convexity · Order continuity · Extreme point · Existence set · One-complemented subspace

Mathematics Subject Classification 46E30 - 46B20 · 46B28

\section{Introduction}

Geometric structures with application of the Lorentz spaces $\Gamma_{p, w}$ and Marcinkiewicz spaces $M_{\phi}$ in case of the nonatomic measure have been investigated extensively by many authors $[3,5,6,12,13]$. In contrast to the nonatomic case, there are only few papers concerning geometric structure of sequence Lorentz and Marcinkiewicz spaces. The first crucial paper devoted to the Marcinkiewicz spaces appeared in 2004 [9], where

Maciej Ciesielski

maciej.ciesielski@put.poznan.pl

Grzegorz Lewicki

grzegorz.lewicki@im.uj.edu.pl

1 Faculty of Electrical Engineering, Institute of Mathematics, Poznań University of Technology, Piotrowo 3A, 60-965 Poznan, Poland

2 Department of Mathematics and Computer Science, Jagiellonian University, Łojasiewicza 6, 30-348 Kraków, Poland 
authors have studied the biduals and order continuous ideals of the Marcinkiewicz spaces for the pure atomic measure. The next significant paper was published in 2009 [10], in which there has been investigated, among others, strict monotonicity, smooth points, and extreme points with application to one-complemented subspaces. For other results concerning the issue devoted to one-complemented subspaces please see, e.g., $[7,8,11]$.

The purpose of this article is to explore geometric properties of the sequence Lorentz spaces $\gamma_{p, w}$ and its dual and predual spaces. It is worth mentioning that we present an application of geometric properties to a characterization of one-complemented subspaces in the Lorentz spaces $\gamma_{p, w}$ in case of the pure atomic measure. It is necessary to mention that a characterization of geometric structure of the sequence Lorentz and Marcinkiewicz spaces does not follow immediately as a consequence of well-known results from the case of nonatomic measure in general.

The paper is organized as follows. In Sect. 2, we present the needed terminology. In Sect. 3, we show an auxiliary result devoted to a relationship between the global convergence in measure of a sequence $\left(x_{n}\right) \subset \ell^{0}$ and the pointwise convergence of its sequence of decreasing rearrangements $\left(x_{n}^{*}\right)$. In case of the pure atomic measure, we also establish a correspondence between an identity of signs of the values for two different sequences in $\ell^{0}$ and an additivity of the decreasing rearrangement operation for these sequences. Section 4 is devoted to an investigation of geometric structure of sequence Lorentz spaces $\gamma_{p}, w$. Namely, we focus on complete criteria for order continuity and the Fatou property in Lorentz spaces for the pure atomic measure. Next, we present a characterization of strict monotonicity and strict convexity of $\gamma_{p}, w$ written in terms of the weight sequence $w$. In spirit of the previous result, we describe an equivalent condition for extreme points of the unit ball in the sequence Lorentz space $\gamma_{1, w}$. In Sect. 5, we solve the essential problem showing a full description of the dual and predual spaces of the sequence Lorentz space $\gamma_{1, w}$. First, we answer a crucial question under which condition does an isometric isomorphism exist between the dual space of the sequence Lorentz space $\gamma_{1, w}$ and the sequence Marcinkiewicz space $m_{\phi}$. Next, we discuss complete criteria which guarantee that the predual space of the sequence Lorentz space $\gamma_{1, w}$ coincides with the sequence Marcinkiewicz space $m_{\phi}^{0}$. Additionally, we investigate necessary condition for the isometry between the predual of $\gamma_{1, w}$ and the Marcinkiewicz space $m_{\phi}^{0}$. In Sect. 5, we present an application of geometric properties of the sequence Lorentz space $\gamma_{1, w}$ to a characterization of one-complemented subspaces. Namely, using an isometry between the classical Lorentz space $d_{1, w}$ and the Lorentz space $\gamma_{1, w}$, we prove that there exists norm one projection on any nontrivial existence subspace of $\gamma_{1, w}$. Additionally, by the previous investigation and in view of [10], we establish a full characterization of smooth points in the sequence Lorentz space $\gamma_{1, w}$ and its predual and dual spaces. Finally, we study an equivalent condition for extreme points in the dual space of the sequence Lorentz space $\gamma_{1, w}$.

\section{Preliminaries}

Let $\mathbb{R}, \mathbb{R}^{+}$, and $\mathbb{N}$ be the sets of reals, nonnegative reals, and positive integers, respectively. A mapping $\phi: \mathbb{N} \rightarrow \mathbb{R}^{+}$is said to be quasiconcave if $\phi(t)$ is increasing and 
$\phi(t) / t$ is decreasing on $\mathbb{N}$ and also $\phi(n)>0$ for all $n \in \mathbb{N}$. Denote by $\ell^{0}$ the set of all real sequences, and by $S_{X}$ (resp. $B_{X}$ ) the unit sphere (resp. the closed unit ball) in a Banach space $\left(X,\|\cdot\|_{X}\right)$. Let us denote by $\left(e_{i}\right)_{i=1}^{\infty}$ a standard basis in $\mathbb{R}^{\infty}$. A quasiBanach lattice $E \subset \ell^{0}$ equipped with a quasi-norm $\|\cdot\|_{E}$ is called a quasi-Banach sequence space (or a quasi-Köthe sequence space) if the following conditions hold

(1) If $x \in \ell^{0}, y \in E$, and $|x| \leq|y|$, then $x \in E$ and $\|x\|_{E} \leq\|y\|_{E}$.

(2) There exists a strictly positive $x \in E$.

For simplicity let us use the short symbol $E^{+}=\{x \in E: x \geq 0\}$. An element $x \in E$ is called a point of order continuity, shortly $x \in E_{a}$, if for any sequence $\left(x_{n}\right) \subset E^{+}$ such that $x_{n} \leq|x|$ and $x_{n} \rightarrow 0$ pointwise we have $\left\|x_{n}\right\|_{E} \rightarrow 0$. A quasi-Banach sequence space $E$ is said to be order continuous, shortly $E \in(\mathrm{OC})$, if any element $x \in E$ is a point of order continuity. Given a quasi-Banach sequence space $E$ is said to have the Fatou property if for all $\left(x_{n}\right) \subset E^{+}, \sup _{n \in \mathbb{N}}\left\|x_{n}\right\|_{E}<\infty$ and $x_{n} \uparrow x \in \ell^{0}$, then $x \in E$ and $\left\|x_{n}\right\|_{E} \uparrow\|x\|_{E}$ (see $[2,16]$ ). We say that $E$ is strictly monotone if for any $x, y \in E^{+}$such that $x \leq y$ and $x \neq y$ we have $\|x\|_{E}<\|y\|_{E}$.

Let $\left(X,\|\cdot\|_{X}\right)$ be a Banach space. Recall that $x \in S_{X}$ is an extreme point of $B_{X}$ if for any $y, z \in S_{X}$ such that $x=(y+z) / 2$ we have $x=y=z$. A Banach space $X$ is called rotund or strictly convex if any $x \in S_{X}$ is an extreme point of $B_{X}$. An element $x \in X$ is called a smooth point of $X$ if there exists a unique linear bounded functional $f \in S_{X^{*}}$ such that $f(x)=\|x\|_{X}$.

The distribution for any sequence $x \in \ell^{0}$ is defined by

$$
d_{x}(\lambda)=\operatorname{card}\{k \in \mathbb{N}:|x(k)|>\lambda\}, \quad \lambda \geq 0 .
$$

For any sequence $x \in \ell^{0}$ its decreasing rearrangement is given by

$$
x^{*}(n)=\inf \left\{\lambda \geq 0: d_{x}(\lambda) \leq n-1\right\}, \quad n \in \mathbb{N} .
$$

In this article we use the notation $x^{*}(\infty)=\lim _{n \rightarrow \infty} x^{*}(n)$. For any sequence $x \in \ell^{0}$ we denote the maximal sequence of $x^{*}$ by

$$
x^{* *}(n)=\frac{1}{n} \sum_{i=1}^{n} x^{*}(i)
$$

It is easy to notice that for any point $x \in \ell^{0}, x^{*} \leq x^{* *}, x^{* *}$ is decreasing and subadditive. For more details of $d_{x}, x^{*}$, and $x^{* *}$ see [2,14].

We say that two sequences $x, y \in \ell^{0}$ are equimeasurable, shortly $x \sim y$, if $d_{x}=$ $d_{y}$. A quasi-Banach sequence space $\left(E,\|\cdot\|_{E}\right)$ is called symmetric or rearrangement invariant (r.i. for short) if whenever $x \in \ell^{0}$ and $y \in E$ such that $x \sim y$, then $x \in E$ and $\|x\|_{E}=\|y\|_{E}$. The fundamental sequence $\phi_{E}$ of a symmetric space $E$ we define as follows $\phi_{E}(n)=\left\|\chi_{\{i \in \mathbb{N}: i \leq n\}}\right\|_{E}$ for any $n \in \mathbb{N}$ (see [2]). Let $0<p<\infty$ and $w=(w(n))_{n \in \mathbb{N}}$ be a nonnegative real sequence and let for any $n \in \mathbb{N}$

$$
W(n)=\sum_{i=1}^{n} w(i) \text { and } W_{p}(n)=n^{p} \sum_{i=n+1}^{\infty} \frac{w(i)}{i^{p}}<\infty .
$$


For short notation the sequence $w$ is called a nonnegative weight sequence. In the whole paper, unless we say otherwise we suppose that $w$ a nonnegative weight sequence is nontrivial, i.e., there is $n \in \mathbb{N}$ such that $w(n)>0$. Let $0<p<\infty$. Now, we recall the sequence Lorentz space $d_{p, w}$ which is a subspace of $\ell^{0}$ such that for any sequence $x=(x(n))_{n \in \mathbb{N}} \in d_{p, w}$ we have

$$
\|x\|_{d_{p, w}}=\left(\sum_{i=1}^{\infty} x^{* p}(n) w(n)\right)^{1 / p}<\infty .
$$

It is well known that the Lorentz space $d_{p, w}$ is a norm space if and only if $w$ is decreasing (see [17]). Furthermore, for any $0<p<\infty,\|\cdot\|_{\gamma_{p, w}}$ is a quasi-norm if and only if $W$ satisfies condition $\Delta_{2}$, that is $W(2 n) \leq K W(n)$ for any $n \in \mathbb{N}$ and for some $K>0$ (see [13,20]). Additionally, if $W$ satisfies condition $\Delta_{2}$ and $W(\infty)=\infty$, then for any $0<p<\infty$ the space $d_{p, w}$ is a separable r.i. quasi-Banach sequence space (see [13]). Recall, the sequence Lorentz space $\gamma_{p, w}$ is a collection of all real sequences $x=(x(n))_{n \in \mathbb{N}}$ such that

$$
\|x\|_{\gamma_{p, w}}=\left(\sum_{i=1}^{\infty}\left(x^{* *}(n)\right)^{p} w(n)\right)^{1 / p}<\infty .
$$

Let us notice that for any nonnegative sequence $w=(w(n))_{n \in \mathbb{N}}$ the sequence Lorentz space $\gamma_{p, w}$ is a r.i. (quasi-)Banach sequence space equipped with the (quasi-)norm $\|\cdot\|_{\gamma_{p, w}}$. Additionally, note that the space $\gamma_{p, w}$ is a Banach space if $1 \leq p<\infty$. It is easy to observe that the fundamental sequence of the Lorentz space $\gamma_{p, w}$ is given by

$$
\phi_{\gamma_{p, w}}(n)=\left\|\chi_{\{i \leq n, i \in \mathbb{N}\}}\right\|_{\gamma_{p, w}}=\left(W(n)+W_{p}(n)\right)^{1 / p}
$$

for every $n \in \mathbb{N}$. Clearly, since $x^{*} \leq x^{* *}$, we have $\gamma_{p, w} \subset d_{p, w}$ for any $0<p<\infty$. Moreover, it is well known that $d_{p, w}=\gamma_{p, w}$ for any $0<p<\infty$ if and only if $w$ satisfies $B_{p}$, i.e., there exists $A>0$ such that for every $n \in \mathbb{N}$ we have $W_{p}(n) \leq$ $A W(n)$ (for more details see [12,13]).

Let $\phi$ be a quasiconcave sequence. The Marcinkiewicz space $m_{\phi}$ and (resp. $m_{\phi}^{0}$ ) consists of all real sequences $x=(x(n))_{n \in \mathbb{N}}$ such that

$$
\|x\|_{m_{\phi}}=\sup _{n \in \mathbb{N}}\left\{x^{* *}(n) \phi(n)\right\}<\infty \quad\left(\text { resp. } m_{\phi}^{0} \subset m_{\phi} \text { and } \lim _{n \rightarrow \infty} x^{* *}(n) \phi(n)=0\right) .
$$

Recall that $m_{\phi}$ and $m_{\phi}^{0}$ are symmetric spaces equipped with the norm $\|\cdot\|_{m_{\phi}}$ (for more details see [9]).

\section{Properties of Decreasing Rearrangement for a Pure Atomic Measure}

In this section, first we present an auxiliary lemma devoted to a correspondence between the global convergence in measure on $\mathbb{N}$ of an arbitrary sequence of ele- 
ments in $\ell^{0}$ to an element in $\ell^{0}$ and the pointwise convergence of their decreasing rearrangements. Although the similar result emerges in case of the nonatomic measure space (see [14]), the proof of it is not valid in case of the pure atomic measure space. It is worth mentioning that in the pure atomic measure space the proof of the wanted result is quite long and requires new techniques.

Lemma 3.1 Let $x_{m}, x \in \ell^{0}$ for all $m \in \mathbb{N}$. Assume that $x_{m}$ converges to $x$ globally in measure, that is for any $\epsilon>0$

$$
\lim _{m \rightarrow \infty} \operatorname{card}\left\{n \in \mathbb{N}:\left|x_{m}(n)-x(n)\right|>\epsilon\right\}=0 .
$$

Then, $x_{m}^{*}(n)$ converges to $x^{*}(n)$ for every $n \in \mathbb{N}$.

Proof Let $\left(x_{m}\right) \subset \ell^{0}, x \in \ell^{0}$ be such that $x_{m} \rightarrow x$ globally in measure. Since for any $\epsilon>0$ and $m \in \mathbb{N}$ we have

$$
\operatorname{card}\left\{n \in \mathbb{N}:\left|x_{m}(n)-x(n)\right|>\epsilon\right\} \geq \operatorname{card}\left\{n \in \mathbb{N}:|| x_{m}(n)|-| x(n) \|>\epsilon\right\},
$$

without loss of generality, we may assume that $x \geq 0$ and $x_{m} \geq 0$ for all $n \in \mathbb{N}$. Let $B=\left\{b_{i}\right\}$ be a set of all values for a function $x: \mathbb{N} \rightarrow \mathbb{R}^{+}$. Define for any $i \in\{1, \ldots, \operatorname{card}(B)\}$,

$$
N_{i}=\left\{n \in \mathbb{N}: x(n)=b_{i}\right\}, \quad \text { and } \quad c_{i}=\sum_{j=1}^{i} \operatorname{card}\left(N_{j}\right), \quad c_{0}=0
$$

Without loss of generality we may assume that $\left(b_{i}\right)$ is strictly decreasing. Now we present the proof in three cases.

Case 1 Suppose that $\operatorname{card}\left(N_{1}\right)=\infty$. Then, it is easy to see that $x^{*}(n)=b_{1} \chi_{\mathbb{N}}$. If $b_{1}=0$ then for all $m \geq M_{\delta_{1}}$ we have

$$
d_{x_{m}}\left(\delta_{1}\right)=\operatorname{card}\left\{n \in \mathbb{N}:\left|x_{m}(n)\right|>\delta_{1}\right\}<1
$$

Hence, since $d_{x_{m}^{*}}\left(\delta_{1}\right)=d_{x_{m}}\left(\delta_{1}\right)$ for every $m \geq M_{\delta_{1}}$, we get $x_{m}^{*} \rightarrow 0$ globally in measure, whence we infer that $x_{m}^{*} \rightarrow 0$ pointwise. In case when $B=\left\{b_{1}\right\}$ then we take $b_{2}=0$. Denote $\delta_{1}=\left(b_{1}-b_{2}\right) / 4$. Since $x_{m} \rightarrow x$ globally in measure, there exists $M_{\delta_{1}} \in \mathbb{N}$ such that for all $m \geq M_{\delta_{1}}$,

$$
\operatorname{card}\left\{n \in \mathbb{N}:\left|x_{m}(n)-x(n)\right|>\delta_{1}\right\}<1
$$

Now, we claim that for any $n \in \mathbb{N}, x_{m}^{*}(n) \rightarrow x^{*}(n)$. Indeed, by (1) we conclude that for any $m \geq M_{\delta_{1}}$ and $n \in \mathbb{N}$,

$$
\left|x(n)-x_{m}(n)\right| \leq \delta_{1} .
$$


If $\operatorname{card}\left(\mathbb{N} \backslash N_{1}\right)=0$, then we are done. Otherwise, for any $n \in N_{1}$ and $k \in \mathbb{N} \backslash N_{1}$ we observe that

$$
x_{m}(n) \geq x(n)-\delta_{1}=b_{1}+\frac{3\left(b_{1}-b_{2}\right)}{4}=b_{1}+3 \delta_{1}>x(k)+3 \delta_{1} \geq x_{m}(k)+2 \delta_{1}
$$

for all $m \geq M_{\delta_{1}}$. Consequently, for every $m \geq M_{\delta_{1}}$ we obtain $x_{m}^{*}=\left(x_{m} \chi_{N_{1}}\right)^{*}$ and also $\left|b_{1}-x_{m}(n)\right| \leq \delta_{1}$ for each $n \in N_{1}$. Therefore, for all $m \geq M_{\delta_{1}}$ and $n \in \mathbb{N}$ it is easy to notice that

$$
\delta_{1} \geq\left|b_{1}-x_{m}^{*}(n)\right|=\left|x^{*}(n)-x_{m}^{*}(n)\right| .
$$

Case 2 Assume that there exists $b_{j_{0}} \in B \backslash\{0\}$ such that $\operatorname{card}\left(N_{j_{0}}\right)=\infty$ and $0<$ $\operatorname{card}\left(N_{j}\right)<\infty$ for any $j \in\left\{1, \ldots, j_{0}-1\right\}$. Then, we have

$$
x^{*}(n)=\left(\sum_{j=1}^{j_{0}} b_{j} \chi_{N_{j}}\right)^{*}(n)=\sum_{j=1}^{j_{0}} b_{j} \chi_{\left\{i \in \mathbb{N}: c_{j-1}+1 \leq i \leq c_{j}\right\}}(n) .
$$

In case when card $(B)=j_{0}$ then we assume that $b_{j_{0}+1}=0$. Denote for any $i \in$ $\{1, \ldots, \operatorname{card}(B)\}$,

$$
\delta_{i}=\frac{b_{i}-b_{i+1}}{4} \text { and } \delta=\min _{1 \leq i \leq j_{0}}\left\{\delta_{i}\right\} .
$$

Since $x_{m} \rightarrow x$ globally in measure, there exists $M_{\delta} \in \mathbb{N}$ such that for all $m \geq M_{\delta}$,

$$
\operatorname{card}\left\{n \in \mathbb{N}:\left|x_{m}(n)-x(n)\right|>\delta\right\}<1 .
$$

Therefore, for any $m \geq M_{\delta}$ and $n_{i} \in N_{i}$ where $1 \leq i \leq j_{0}$ we have

$$
\delta \geq\left|x\left(n_{i}\right)-x_{m}\left(n_{i}\right)\right|=\left|b_{i}-x_{m}\left(n_{i}\right)\right| .
$$

Hence, for all $m \geq M_{\delta}$ and $n_{i} \in N_{i}$ where $1 \leq i \leq j_{0}-1$ we easily observe

$$
x_{m}\left(n_{i}\right)=b_{i}-\delta \geq b_{i+1}+3 \delta \geq x_{m}\left(n_{i+1}\right)+2 \delta .
$$

In consequence, by (3) we get for every $m \geq M_{\delta}$ and $n \in \mathbb{N}$,

$$
x_{m}^{*}(n)=\left(\sum_{j=1}^{j_{0}} x_{m} \chi_{N_{j}}\right)^{*}(n)=\sum_{j=1}^{j_{0}}\left(x_{m} \chi_{N_{j}}\right)^{*}\left(n-c_{j-1}\right) \chi_{\left\{i \in \mathbb{N}: c_{j-1}+1 \leq i \leq c_{j}\right\}}(n)
$$

Clearly, there exists $\sigma: \mathbb{N} \rightarrow \bigcup_{j=1}^{j_{0}} N_{j}$ a permutation such that $x^{*}(n)=x(\sigma(n))$ for all $n \in \mathbb{N}$. Thus, for any $n \in \mathbb{N}$ there exists $j \in\left\{1, \ldots, j_{0}\right\}$ such that $\sigma(n) \in N_{j}$ and by (3) we obtain 


$$
\delta \geq\left|x_{m}(\sigma(n))-x(\sigma(n))\right|=\left|x_{m}(\sigma(n))-b_{j}\right|=\left|\left(x_{m} \chi_{N_{j}}\right)^{*}\left(n-c_{j-1}\right)-b_{j}\right|
$$

for all $m \geq M_{\delta}$. Therefore, by (2) and (4) we infer that

$$
\begin{aligned}
x_{m}^{*}(n) & =\sum_{j=1}^{j_{0}}\left(x_{m} \chi_{N_{j}}\right)^{*}\left(n-c_{j-1}\right) \chi_{\left\{i \in \mathbb{N}: c_{j-1}+1 \leq i \leq c_{j}\right\}}(n) \\
& \rightarrow \sum_{j=1}^{j_{0}} b_{j} \chi_{\left\{i \in \mathbb{N}: c_{j-1}+1 \leq i \leq c_{j}\right\}}(n)=x^{*}(n) .
\end{aligned}
$$

Case 3 Suppose that for any $b_{j} \in B \backslash\{0\}$ we have $\operatorname{card}\left(N_{j}\right)<\infty$. If $\operatorname{card}(B)<\infty$ then without loss of generality we may assume that $j_{0}=\operatorname{card}(B)$ and $b_{j_{0}}=0$. Next, letting for any $i \in\left\{1, \ldots, j_{0}-1\right\}$,

$$
\delta_{i}=\frac{b_{i}-b_{i+1}}{4} \text { and } \delta=\min _{1 \leq i \leq j_{0}-1}\left\{\delta_{i}\right\}
$$

and proceeding analogously as in case 2 we may show that $x_{m}^{*} \rightarrow x^{*}$ on $\mathbb{N}$, in case when $\operatorname{card}(B)<\infty$. Now, assume that $\operatorname{card}(B)=\infty$. Then, since $\left(b_{j}\right)$ is strictly decreasing and bounded we conclude

$$
\lim _{j \rightarrow \infty} b_{j}=b \geq 0
$$

First, let us consider that $b=0$. Let $\epsilon>0$. Then, there exists $j_{0} \in \mathbb{N}$ such that for all $j \geq j_{0}$ we have

$$
0<b_{j}<\frac{\epsilon}{4} \text { and } \quad b_{j_{0}-1} \geq \frac{\epsilon}{4} .
$$

Define for any $i \in\left\{1, \ldots, j_{0}\right\}$,

$$
\delta_{i}=\frac{b_{i}-b_{i+1}}{4} \text { and } \delta=\min \left\{\frac{\epsilon / 4-b_{j_{0}}}{4}, \min _{1 \leq i \leq j_{0}}\left\{\delta_{i}\right\}\right\} .
$$

Similarly as in case 2 there is $M_{\delta} \in \mathbb{N}$ such that for all $m \geq M_{\delta}, n \in \mathbb{N}$, and $k \in \bigcup_{j \geq j_{0}} N_{j}$ we get

$$
\left|x_{m}(n)-x(n)\right| \leq \delta \quad \text { and } \quad x_{m}(k) \leq \delta+x(k) \leq \delta+b_{j_{0}}<\delta+\frac{\epsilon}{4}<\frac{\epsilon}{2} .
$$

Moreover, we may observe that

$$
x_{m}\left(n_{i}\right) \geq x_{m}\left(n_{i+1}\right)+2 \delta
$$

for every $m \geq M_{\delta}$ and $n_{i} \in N_{i}$ where $i \in\left\{1, \ldots, j_{0}-1\right\}$. Next, assuming that $\sigma: \mathbb{N} \rightarrow \bigcup_{j=1}^{\infty} N_{j}$ is a permutation such that $x^{*}(n)=x(\sigma(n))$ for all $n \in \mathbb{N}$, then 
for any $n \in \mathbb{N}$ with $n \leq c_{j_{0}-1}$ there exists $j \in\left\{1, \ldots, j_{0}-1\right\}$ such that $\sigma(n) \in N_{j}$ and by (6) we obtain

$$
\begin{aligned}
\epsilon>\delta & \geq\left|x_{m}(\sigma(n))-x(\sigma(n))\right|=\left|x_{m}(\sigma(n))-b_{j}\right| \\
& =\left|\left(x_{m} \chi_{N_{j}}\right)^{*}\left(n-c_{j-1}\right)-b_{j}\right|=\left|\left(\sum_{j=1}^{j_{0}-1} x_{m} \chi_{N_{j}}\right)^{*}(n)-b_{j}\right|
\end{aligned}
$$

for all $m \geq M_{\delta}$. On the other hand, if $n>c_{j_{0}-1}$ then there is $j \geq j_{0}$ such that $\sigma(n) \in N_{j}$ and by (5) and (6) it follows that

$$
\begin{aligned}
\left|\left(\sum_{j=j_{0}}^{\infty} x_{m} \chi_{N_{j}}\right)^{*}\left(n-c_{j_{0}-1}\right)-x^{*}(n)\right| & =\left|x_{m}(\sigma(n)) \chi_{N_{j}}(\sigma(n))-x(\sigma(n))\right| \\
& =\left|x_{m}(\sigma(n))-b_{j}\right|<\epsilon
\end{aligned}
$$

for all $m \geq M_{\delta}$. Now, let us notice that for every $n \in \mathbb{N}$,

$$
x^{*}(n)=\sum_{j=1}^{\infty} b_{j} \chi_{\left\{i \in \mathbb{N}: c_{j-1}+1 \leq i \leq c_{j}\right\}}(n)
$$

and

$$
x_{m}^{*}(n)= \begin{cases}\left(\sum_{j=1}^{j_{0}-1} x_{m} \chi_{N_{j}}\right)^{*}(n) & \text { if } n \leq c_{j_{0}-1}, \\ \left(\sum_{j=j_{0}}^{\infty} x_{m} \chi_{N_{j}}\right)^{*}\left(n-c_{j_{0}-1}\right) & \text { if } n>c_{j_{0}-1} .\end{cases}
$$

Hence, we infer that for any $m \geq M_{\delta}$ and $n \in \mathbb{N}$,

$$
x_{m}^{*}(n) \rightarrow x^{*}(n)
$$

Now, we assume that $b>0$. Then, it is easy to see that $x^{*}(\infty)=b>0$. Next, taking

$$
y=x \chi_{\operatorname{supp}(x)}+b \chi_{\mathbb{N} \backslash \operatorname{supp}(x)} \text { and } \quad y_{m}=x_{m} \chi_{\operatorname{supp}(x)}+b \chi_{\mathbb{N} \backslash \operatorname{supp}(x)}
$$

for all $m \in \mathbb{N}$, we may show that $x^{*}=y^{*}$ and $x_{m}^{*}=y_{m}^{*}$ for sufficiently large $m \in \mathbb{N}$. Next, passing to subsequence and relabeling if necessary, it is enough to prove that $y_{m}^{*} \rightarrow y^{*}$ on $\mathbb{N}$. Clearly, by definition of $y$ and $y_{m}$ for all $m \in \mathbb{N}$ we may observe that $y_{m}-b$ converges $y-b$ globally in measure and $(y-b)^{*}(\infty)=0$. Finally, using analogous technique as previously, in case 3 for $b=0$, we finish the proof.

Remark 3.2 Let us notice that using analogous techniques as in the proof in [14, property $9^{0}$ ] and by the property $7^{0}$ in [14] (see pp. 64-65), in view of [2, Theorem 2.7 and Proposition 3.3] we are able to show the below assertion.

For any two sequences $x$ and $y$ with $x^{*}(\infty)=0$ and $y^{*}(\infty)=0$ the following conditions are equivalent. 
(i) For any $i \in \mathbb{N}$,

$$
(x+y)^{*}(i)=x^{*}(i)+y^{*}(i) .
$$

(ii) $\operatorname{sgn}(x(i))=\operatorname{sgn}(y(i))$ for any $i \in \mathbb{N}$ and there exists $\left(E_{n}\right)_{n \in \mathbb{N}}$ a countable collection of subsets of $\mathbb{N}$ such that for every $n \in \mathbb{N}$ we have $\operatorname{card}\left(E_{n}\right)=n$ and

$$
x^{* *}(n)=\frac{1}{n} \sum_{i \in E_{n}}|x(i)| \text { and } y^{* *}(n)=\frac{1}{n} \sum_{i \in E_{n}}|y(i)| .
$$

\section{Geometric Structure of Sequence Lorentz Spaces $\gamma_{p, w}$}

In this section, we discuss complete criteria for order continuity, the Fatou property, strict monotonicity and strict convexity, and also extreme points of the unit ball in the sequence Lorentz space $\gamma_{p, w}$.

Theorem 4.1 Let $w$ be a nonnegative weight sequence and $0<p<\infty$. The Lorentz space $\gamma_{p, w}$ is order continuous if and only if $W(\infty)=\infty$.

Proof Necessity Suppose that $\gamma_{p, w}$ is not order continuous. Then, there exists $\left(x_{m}\right) \subset$ $\gamma_{p, w}^{+} \backslash\{0\}$ such that $x_{m} \downarrow 0$ pointwise and $d=\inf _{n \in \mathbb{N}}\left\|x_{m}\right\|_{\gamma_{p, w}}>0$. Next, passing to subsequence and relabeling if necessary we may assume that $\left\|x_{m}\right\|_{\gamma_{p, w}} \downarrow d$. Since $W(\infty)=\infty$ we claim that $d_{x}(\lambda)<\infty$ for all $\lambda>0$ and $x \in \gamma_{p, w}$. Indeed, assuming for a contrary that there is $x \in \gamma_{p, w}$ such that $x^{*}(\infty)=\lim _{n \rightarrow \infty} x^{*}(n)>0$ we obtain $\ell^{\infty} \hookrightarrow \gamma_{p, w}$. Define $z=\chi_{\mathbb{N}}$. Then, we have $z^{* *}=z \in \gamma_{p, w}$ and also $\|z\|_{\gamma_{p, w}}=W(\infty)=\infty$, which gives us a contradiction and proves the claim. Let $\epsilon>0$. Define two sets

$$
E_{1}=\left\{n \in \mathbb{N}: x_{1}(n)>\epsilon\right\} \text { and } E_{2}=\mathbb{N} \backslash E_{1}
$$

Now, since $x_{1}^{*}(\infty)=0$ it is easy to notice $\operatorname{card}\left(E_{1}\right)=d_{x_{1}}(\epsilon)<\infty$ and $E_{1} \cap E_{2}=\emptyset$. Therefore, since $x_{m} \downarrow 0$ pointwise we have

$$
d_{x_{m}}(\epsilon)=\operatorname{card}\left\{n \in \mathbb{N}: x_{m}(n)>\epsilon\right\} \rightarrow 0 \text { as } m \rightarrow \infty .
$$

Hence, by Lemma 3.1 it follows that $x_{m}^{*} \rightarrow 0$ pointwise on $\mathbb{N}$. Consequently, since $\left\|x_{1}\right\|_{\gamma_{p, w}}<\infty$ and $x^{* *}(n)<\infty$ for all $n \in \mathbb{N}$, applying twice the Lebesgue Dominated Convergence Theorem we conclude $\left\|x_{m}\right\|_{\gamma_{p, w}} \rightarrow 0$.

Sufficiency Assume for a contrary that $W(\infty)<\infty$. Then, it is easy to see that $x=\chi_{\mathbb{N}} \in \gamma_{p, w}, x^{* *}=x$, and $\|x\|_{\gamma_{p, w}}=W(\infty)$. Define $x_{m}=\chi_{\{i \in \mathbb{N}: i \geq m\}}$ for any $m \in \mathbb{N}$. Clearly, we have $x_{m} \downarrow 0$ and $x_{m} \leq x$ pointwise for every $m \in \mathbb{N}$. Moreover, we observe that $x_{m}^{* *}=x^{* *}$ for any $m \in \mathbb{N}$. Hence, we get $\left\|x_{m}\right\|_{\gamma_{p, w}}=W(\infty)>0$ for all $n \in \mathbb{N}$, which contradicts with assumption that $\gamma_{p, w}$ is order continuous. 
Remark 4.2 First, let us recall that for any symmetric Banach function space $X$ over $[0, \infty)$ with the fundamental function $\phi_{X}$ we have $X \hookrightarrow M_{\phi_{X}}$ and the embedding has norm 1 , where

$$
M_{\phi}=\left\{x: \sup _{t>0}\left\{\frac{\phi_{X}(t)}{t} \int_{0}^{t} x^{*}(s) \mathrm{d} s\right\}<\infty\right\}
$$

(for more details see [2, Proposition 5.9]). Now, observe that for any symmetric Banach sequence space $E$ over $\mathbb{N}$, in [2, Proposition 5.9] is also satisfied. Namely, using analogous technique as in [2] we are able to show that for any symmetric Banach sequence space $E$ the embedding $E \hookrightarrow m_{\phi}$ holds with constant 1, i.e., for all $x \in E$, we have

$$
\|x\|_{m_{\phi}}=\sup \left\{x^{* *}(n) \phi_{E}(n)\right\} \leq\|x\|_{E},
$$

where $\phi_{E}$ is the fundamental sequence of $E$ on $\mathbb{N}$. Next, assuming that $E$ a symmetric Banach sequence space with the Fatou property, in view of [4, Remark 3.2] we obtain that $\phi_{E}(\infty)=\infty$ if and only if $x^{*}(\infty)=0$ for any $x \in E$.

Lemma 4.3 Let $w$ be a nonnegative weight sequence and $0<p<\infty$. The Lorentz space $\gamma_{p, w}$ has the Fatou property.

Proof Let $\left(x_{m}\right) \subset \gamma_{p, w}^{+}, x \in \ell^{0}$ and $x_{m} \uparrow x$ pointwise and $\sup _{m \in \mathbb{N}}\left\|x_{m}\right\|_{\gamma_{p, w}}<\infty$. Immediately, by [2, Proposition 1.7] it follows that $x_{m}^{*} \uparrow x^{*}$. Next, applying twice Lebesgue Monotone Convergence Theorem [19] we get $\left\|x_{m}\right\|_{\gamma_{p, w}} \uparrow\|x\|_{\gamma_{p, w}}$. Finally, since $\sup _{m \in \mathbb{N}}\left\|x_{m}\right\|_{\gamma_{p, w}}<\infty$ it follows that $x \in \gamma_{p, w}$.

Theorem 4.4 Let $w$ be a nonnegative weight sequence and $0<p<\infty$. The Lorentz space $\gamma_{p, w}$ is strictly monotone if and only if $W(\infty)=\infty$.

Proof Necessity Assume for a contrary that $W(\infty)<\infty$. Then, we may show that $\ell^{\infty} \hookrightarrow \gamma_{p, w}$. Next, defining two sequences

$$
x=\chi_{\{i \in \mathbb{N}: i>1\}} \text { and } y=\chi_{\mathbb{N}}
$$

we easily observe that $x \leq y, x \neq y$, and $x^{* *}=y^{* *}=y$. Consequently, $\|x\|_{\gamma_{p, w}}=$ $\|y\|_{\gamma_{p, w}}$, which contradicts with assumption that the Lorentz space $\gamma_{p, w}$ is strictly monotone.

Sufficiency Let $x, y \in \gamma_{p, w}^{+}, x \leq y$, and $x \neq y$. Since $x \neq y$ there exists $n_{0} \in \mathbb{N}$ such that $x\left(n_{0}\right)<y\left(n_{0}\right)$. Define

$$
\delta_{0}=\max \left\{y\left(n_{0}\right) / 2, x\left(n_{0}\right)\right\} \text { and } N_{0}=\left\{n \in \mathbb{N}: y(n)>\delta_{0}\right\} .
$$

Since $W(\infty)=\infty$, by the proof of Theorem 4.1 it follows that $y^{*}(\infty)=x^{*}(\infty)=0$. Hence, since $n_{0} \in N_{0}$ we get

$$
0<\operatorname{card}\left(N_{0}\right)=d_{y}\left(\delta_{0}\right)<\infty .
$$


Now, we claim that there exists $m_{0} \in\left\{1, \ldots, \operatorname{card}\left(N_{0}\right)\right\}$ such that $x^{*}\left(m_{0}\right)<y^{*}\left(m_{0}\right)$. Indeed, if it is not true then for all $n \in\left\{1, \ldots, \operatorname{card}\left(N_{0}\right)\right\}$ we have $x^{*}(n)=y^{*}(n)$. Moreover, there is a permutation $\sigma: \mathbb{N} \rightarrow \mathbb{N}$ such that $\sigma(n) \in N_{0}$ and $y^{*}(n)=$ $y(\sigma(n))$ for every $n \in\left\{1, \ldots, \operatorname{card}\left(N_{0}\right)\right\}$. So, we have

$$
x^{*}(n)=y^{*}(n)=y(\sigma(n)) \geq x(\sigma(n))
$$

for any $n \in\left\{1, \ldots, \operatorname{card}\left(N_{0}\right)\right\}$. Let $m_{0} \in\left\{1, \ldots, \operatorname{card}\left(N_{0}\right)\right\}$ be such that $\sigma\left(m_{0}\right)=n_{0}$. Then, we observe that

$$
x^{*}\left(m_{0}\right)=y^{*}\left(m_{0}\right)=y\left(\sigma\left(m_{0}\right)\right)=y\left(n_{0}\right)>x\left(n_{0}\right) .
$$

Therefore, we obtain

$$
x^{*}\left(m_{0}\right)>x\left(n_{0}\right)=x\left(\sigma\left(m_{0}\right)\right),
$$

which implies that there exists $k_{0} \in \mathbb{N} \backslash N_{0}$ such that $x\left(k_{0}\right)=y\left(n_{0}\right)$. On the other hand, it is well known that $x\left(k_{0}\right) \leq y\left(k_{0}\right)$, whence

$$
y\left(k_{0}\right) \geq x\left(k_{0}\right)=y\left(n_{0}\right)=y^{*}\left(m_{0}\right)
$$

In consequence, by definition of $N_{0}$ this yields that $k_{0} \in N_{0}$ and gives us a contradiction. Now, since $x^{*}(n) \leq y^{*}(n)$ for any $n \in \mathbb{N}$ and $x^{*}\left(n_{0}\right)<y^{*}\left(n_{0}\right)$ for some $n_{0} \in \mathbb{N}$ it follows that

$$
x^{* *}(n) \leq y^{* *}(n) \text { and } \sum_{i=1}^{k} x^{*}(i)<\sum_{i=1}^{k} y^{*}(i)
$$

for all $n \in \mathbb{N}$ and $k \geq n_{0}$. Finally, by assumption that $W(\infty)=\infty$ there exists $\left(n_{k}\right) \subset$ $\mathbb{N}$ such that $w\left(n_{k}\right)>0$ for every $k \in \mathbb{N}$. Hence, we infer that $\|x\|_{\gamma_{p, w}}<\|y\|_{\gamma_{p, w}}$.

The immediate consequence of the previous theorem and Proposition 2.1 in [10] is the following result.

Corollary 4.5 Let $w \geq 0$ be a weight sequence such that $W(\infty)=\infty$ and let $1 \leq p<$ $\infty$. An element $x \in S_{\gamma_{p, w}}$ is an extreme point of $B_{\gamma_{p, w}}$ if and only if $x^{*}$ is an extreme point of $B_{\gamma_{p, w}}$.

Next, we show that the Lorentz space $\gamma_{p, w}$ is strictly convex for $1<p<\infty$ and $w$ a positive weight sequence such that $W(\infty)=\infty$. In some parts of the proof of the following theorem, we use the similar techniques to [6, Theorem 3.1] (see also [5, Theorem 2.3]). For the sake of completeness and reader's convenience we show all details of the proof.

Theorem 4.6 Let $w$ be a nonnegative weight sequence. The Lorentz space $\gamma_{p, w}$ is strictly convex if and only if $1<p<\infty$ and $w(n)>0$ for any $n \in \mathbb{N}$ and also $W(\infty)=\infty$. 
Proof Necessity Assume that $\gamma_{p, w}$ is strictly convex. For a contrary we suppose that $p=1$. Let $x, y \in S_{\gamma_{p, w}}$, and $\|x+y\|_{\gamma_{p, w}}=2$. Without loss of generality we may assume that $x=x^{*}$ and $y=y^{*}$. Then, we have $(x+y)^{* *}=x^{* *}+y^{* *}$ and also

$$
\|x+y\|_{\gamma_{p, w}}=\|x\|_{\gamma_{p, w}}+\|y\|_{\gamma_{p, w}}=2
$$

Consequently, since $x$ and $y$ are arbitrary and $\gamma_{p, w}$ is strictly convex we conclude a contradiction. Now, assume that $W(\infty)<\infty$. Define

$$
x=\frac{1}{W(\infty)^{1 / p}} \chi_{\{2 n: n \in \mathbb{N}\}} \text { and } y=\frac{1}{W(\infty)^{1 / p}} \chi_{\mathbb{N}} .
$$

Clearly, we have for any $n \in \mathbb{N}$,

$$
x^{* *}(n)=y^{* *}(n)=\frac{1}{W(\infty)^{1 / p}} .
$$

Moreover, we observe that

$$
(x+y)^{* *}(n)=\frac{1}{W(\infty)^{1 / p}}\left(2 \chi_{\{2 n: n \in \mathbb{N}\}}+\chi_{\{2 n-1: n \in \mathbb{N}\}}\right)^{* *}(n)=\frac{2}{W(\infty)^{1 / p}}
$$

for any $n \in \mathbb{N}$. Hence, we get

$$
\|x\|_{\gamma_{p, w}}=\|y\|_{\gamma_{p, w}}=\frac{\|x+y\|_{\gamma_{p, w}}}{2}=1
$$

Therefore, by assumption that $\gamma_{p, w}$ is strictly convex we obtain a contradiction. Now, let us suppose for a contrary that there is $n_{0} \in \mathbb{N}$ such that $w\left(n_{0}\right)=0$. If $n_{0}=1$, then take $\epsilon \in\left(0,1 / \phi_{\gamma_{p, w}}(2)\right)$ and define

$$
x=\frac{1}{\phi_{\gamma_{p, w}}(2)} \chi_{\{1,2\}} \text { and } y=\left(\frac{1}{\phi_{\gamma_{p, w}}(2)}+\epsilon\right) \chi_{\{1\}}+\left(\frac{1}{\phi_{\gamma_{p, w}}(2)}-\epsilon\right) \chi_{\{2\}} \text {. }
$$

It is easy to see that $x \neq y$ and

$$
x^{* *}(n)=\frac{1}{\phi_{\gamma_{p, w}}(2)} \chi_{\{1,2\}}(n)+\frac{2}{n \phi_{\gamma_{p, w}}(2)} \chi_{\mathbb{N} \backslash\{1,2\}}(n)
$$

and also

$$
y^{* *}(n)=\left(\frac{1}{\phi_{\gamma_{p, w}}(2)}+\epsilon\right) \chi_{\{1\}}(n)+\frac{1}{\phi_{\gamma_{p, w}}(2)} \chi_{\{2\}}(n)+\frac{2}{n \phi_{\gamma_{p, w}}(2)} \chi_{\mathbb{N} \backslash\{1,2\}}(n) .
$$


Therefore, since $w(1)=0$, we have

$$
\begin{aligned}
\|x\|_{\gamma_{p, w}} & =\|y\|_{\gamma_{p, w}}=\left(\frac{1}{\left(\phi_{\gamma_{p, w}}(2)\right)^{p}} w(2)+\frac{2^{p}}{\left(\phi_{\gamma_{p, w}}(2)\right)^{p}} \sum_{n=3}^{\infty} \frac{w(n)}{n^{p}}\right)^{1 / p} \\
& =\frac{1}{\phi_{\gamma_{p, w}}(2)}\left(W(2)+W_{p}(2)\right)^{1 / p}=1
\end{aligned}
$$

Furthermore, we observe that

$$
\begin{aligned}
(x+y)^{* *}(n) & =\left(\left(\frac{2}{\phi_{\gamma_{p, w}}(2)}+\epsilon\right) \chi_{\{1\}}+\left(\frac{2}{\phi_{\gamma_{p, w}}(2)}-\epsilon\right) \chi_{\{2\}}\right)^{* *}(n) \\
& =\left(\frac{2}{\phi_{\gamma_{p, w}}(2)}+\epsilon\right) \chi_{\{1\}}(n)+\frac{4}{n \phi_{\gamma_{p, w}}(2)} \chi_{\mathbb{N} \backslash\{1\}}(n) .
\end{aligned}
$$

Hence, since $w(1)=0$, we get

$$
\begin{aligned}
\|x+y\|_{\gamma_{p, w}} & =\left(\frac{4^{p}}{\left(\phi_{\gamma_{p, w}}(2)\right)^{p}} \sum_{n=2}^{\infty} \frac{w(n)}{n^{p}}\right)^{1 / p}=\frac{2}{\phi_{\gamma_{p, w}}(2)}\left(w(2)+2^{p} \sum_{n=3}^{\infty} \frac{w(n)}{n^{p}}\right)^{1 / p} \\
& =\frac{2}{\phi_{\gamma_{p, w}}(2)}\left(W(2)+W_{p}(2)\right)^{1 / p}=2 .
\end{aligned}
$$

So, in case when $w(1)=0$, it follows that $\gamma_{p, w}$ is not strictly convex. Assume that $n_{0}>1$. Define

$$
x=\frac{1}{\phi_{\gamma_{p, w}}\left(n_{0}\right)} \chi_{\left[1, n_{0}\right]} \text { and } y=\frac{1}{\phi_{\gamma_{p, w}}\left(n_{0}\right)}\left(\chi_{\left[1, n_{0}-1\right]}+\frac{1}{2} \chi_{\left\{n_{0}, n_{0}+1\right\}}\right) .
$$

Then, we easily observe that $x \neq y$ and $\|x\|_{\gamma_{p, w}}=1$. Moreover, we have

$$
y^{* *}(n)=\frac{1}{\phi_{\gamma_{p, w}}\left(n_{0}\right)} \begin{cases}1 & \text { if } n<n_{0}, \\ \frac{n_{0}-1 / 2}{n_{0}} & \text { if } n=n_{0}, \\ \frac{n_{0}}{n} & \text { if } n>n_{0}\end{cases}
$$

and

$$
\begin{aligned}
(x+y)^{* *}(n) & =\frac{1}{\phi_{\gamma_{p, w}}\left(n_{0}\right)}\left(2 \chi_{\left[1, n_{0}-1\right]}+\frac{3}{2} \chi_{\left\{n_{0}\right\}}+\frac{1}{2} \chi_{\left\{n_{0}+1\right\}}\right)^{* *}(n) \\
& =\frac{2}{\phi_{\gamma_{p, w}}\left(n_{0}\right)} \begin{cases}1 & \text { if } n<n_{0}, \\
\frac{n_{0}-1 / 4}{n_{0}} & \text { if } n=n_{0}, \\
\frac{n_{0}}{n} & \text { if } n>n_{0} .\end{cases}
\end{aligned}
$$


Hence, since $w\left(n_{0}\right)=0$, we conclude that

$$
\|y\|_{\gamma_{p, w}}=\frac{\|x+y\|_{\gamma_{p, w}}}{2}=\frac{1}{\phi_{\gamma_{p, w}}\left(n_{0}\right)}\left(W\left(n_{0}-1\right)+W_{p}\left(n_{0}\right)\right)^{1 / p}=1 .
$$

In consequence, by assumption that $\gamma_{p, w}$ is strictly convex we get a contradiction.

Sufficiency Let $x, y \in S_{\gamma_{p, w}}$ and $x \neq y$. We consider the proof in two cases.

Case 1 Assume that there exists $n_{0} \in \mathbb{N}$ such that $x^{* *}\left(n_{0}\right) \neq y^{* *}\left(n_{0}\right)$. Then, by strict convexity of the power function $u^{p}$ for $1<p<\infty$ we have

$$
\left(\frac{1}{2} x^{* *}\left(n_{0}\right)+\frac{1}{2} y^{* *}\left(n_{0}\right)\right)^{p}<\frac{1}{2} x^{* * p}\left(n_{0}\right)+\frac{1}{2} y^{* * p}\left(n_{0}\right) .
$$

Therefore, since for any $n \in \mathbb{N}$,

$$
\left(\frac{1}{2} x^{* *}(n)+\frac{1}{2} y^{* *}(n)\right)^{p} \leq \frac{1}{2} x^{* * p}(n)+\frac{1}{2} y^{* * p}(n)
$$

by assumption that $w(n)>0$ for all $n \in \mathbb{N}$ we infer that $\|x+y\|_{\gamma_{p, w}}<2$.

Case 2 Suppose that $x^{* *}(n)=y^{* *}(n)$ for every $n \in \mathbb{N}$. Thus, we have $x^{*}(n)=y^{*}(n)$ for any $n \in \mathbb{N}$. We claim that there exists $n_{0} \in \mathbb{N}$ such that

$$
(x+y)^{* *}\left(n_{0}\right)<x^{* *}\left(n_{0}\right)+y^{* *}\left(n_{0}\right) .
$$

Indeed, assuming that it is not true it follows that $(x+y)^{*}(n)=x^{*}(n)+y^{*}(n)$ for all $n \in \mathbb{N}$. Consequently, since $W(\infty)=\infty$, by Remark 3.2 we obtain $|x+y|(n)=$ $|x(n)|+|y(n)|$ for all $n \in \mathbb{N}$ and there exists $\left(E_{n}\right)$ an increasing sequence of sets such that $\operatorname{card}\left(E_{n}\right)=n$ for every $n \in \mathbb{N}$ and also

$$
\sum_{i \in E_{n}}|x(i)|=\sum_{i=1}^{n} x^{*}=\sum_{i=1}^{n} y^{*}=\sum_{i \in E_{n}}|y(i)| \text {. }
$$

In consequence, $|x(n)|=|y(n)|$ for any $n \in \mathbb{N}$ and so $x(n)=y(n)$ for every $n \in \mathbb{N}$. Therefore, in view of assumption $x \neq y$ we get a contradiction. Finally, applying the triangle inequality for the maximal function we infer that

$$
\left\|\frac{x+y}{2}\right\|_{\gamma_{p, w}}^{p}<\frac{1}{2}\|x\|_{\gamma_{p, w}}^{p}+\frac{1}{2}\|y\|_{\gamma_{p, w}}^{p}=1 .
$$

Finally, we present a complete criteria for an extreme point in the ball of the Lorentz space $\gamma_{1, w}$. It is worth mentioning that in some parts of the proof we use similar technique to the proof in [10, Theorem 2.6]. For the sake of completeness and reader's convenience, we present all details of the proof of the following theorem. 
Theorem 4.7 Let $w \geq 0$ be a weight sequence such that $W(\infty)=\infty$. An element $x \in S_{\gamma_{1, w}}$ is an extreme point of $B_{\gamma_{1, w}}$ if and only if there exists $n_{0} \in \mathbb{N}$ such that

$$
x^{*}=\frac{1}{\phi_{\gamma_{1, w}}\left(n_{0}\right)} \chi_{\left\{i \in \mathbb{N}: i \leq n_{0}\right\}}
$$

and in case when $n_{0}>1, W\left(n_{0}-1\right)>0$.

Proof Letting $x \in S_{\gamma_{1, w}}$, by Corollary 4.5 we may consider that $x=x^{*}$ is an extreme point of $B_{\gamma_{1, w}}$. Denote

$$
n_{0}=\sup \left\{n \in \mathbb{N}: x^{*}(n)=x^{*}(1)\right\}
$$

Since $W(\infty)=\infty$ and $\phi_{\gamma_{1, w}}(n)=W(n)+W_{1}(n)$ for any $n \in \mathbb{N}$, by Lemma 4.3 and by Remark 4.2 it follows that $x^{*}(\infty)=0$ and so $n_{0} \in \mathbb{N}$. We claim that $x^{*}\left(n_{0}+1\right)=0$. Suppose on the contrary that $x^{*}\left(n_{0}+1\right)>0$ and denote

$$
n_{1}=\operatorname{card}\left\{n \in \mathbb{N}: x^{*}(n)=x^{*}\left(n_{0}+1\right)\right\}
$$

and

$$
d=\min \left\{x^{*}(1)-x^{*}\left(n_{0}+1\right), x^{*}\left(n_{0}+1\right)-x^{*}\left(n_{0}+n_{1}+1\right)\right\}
$$

First, notice that $\phi_{\gamma_{1, w}}(n+1)>\phi_{\gamma_{1, w}}(n)>0$ for any $n \in \mathbb{N}$. Indeed, since $W(\infty)=\infty$ we infer that $\phi_{\gamma_{1, w}}(n)>0$ for all $n \in \mathbb{N}$. Now, assuming for a contrary that there is $n \in \mathbb{N}$ such that $\phi_{\gamma_{1, w}}(n+1)=\phi_{\gamma_{1, w}}(n)$, we easily obtain

$$
w(n+1)=-(n+1) \sum_{i=n+2}^{\infty} \frac{w(i)}{i}<0 .
$$

Hence, since $w(n+1) \geq 0$ we get a contradiction. Now, we are able to find $a, b \in$ $(0, d)$ such that

$$
b=a \frac{\phi_{\gamma_{1, w}}\left(n_{0}+n_{1}\right)-\phi_{\gamma_{1, w}}\left(n_{0}\right)}{\phi_{\gamma_{1, w}}\left(n_{0}\right)} .
$$

Define

$$
y=x^{*}-b \chi_{\left\{i \in \mathbb{N}: i \leq n_{0}\right\}}+a \chi\left\{i \in \mathbb{N}: n_{0}<i \leq n_{0}+n_{1}\right\}
$$

and

$$
z=x^{*}+b \chi\left\{i \in \mathbb{N}: i \leq n_{0}\right\}-a \chi\left\{i \in \mathbb{N}: n_{0}<i \leq n_{0}+n_{1}\right\} .
$$

Clearly, $y \neq z$ and $x=(y+z) / 2$. Since $y=y^{*}$ and $z=z^{*}$, by (8) we have 


$$
\begin{aligned}
\|y\|_{\gamma_{1, w}}= & \sum_{n=1}^{\infty} y^{* *}(n) w(n) \\
= & \sum_{n=1}^{\infty} \frac{w(n)}{n} \sum_{j=1}^{n}\left(x^{*}(j)-b \chi_{\left\{i \in \mathbb{N}: i \leq n_{0}\right\}}(j)+a \chi_{\left\{i \in \mathbb{N}: n_{0}<i \leq n_{0}+n_{1}\right\}}(j)\right) \\
= & \sum_{n=1}^{\infty} x^{* *}(n) w(n)-b\left(\sum_{n=1}^{n_{0}} w(n)+n_{0} \sum_{n=n_{0}+1}^{\infty} \frac{w(n)}{n}\right) \\
& +a\left(\sum_{n=n_{0}+1}^{n_{0}+n_{1}} \frac{w(n)}{n}\left(n-n_{0}\right)+n_{1} \sum_{n=n_{0}+n_{1}+1}^{\infty} \frac{w(n)}{n}\right) \\
= & \|x\|_{\gamma_{1, w}}-b \phi_{\gamma_{1, w}}\left(n_{0}\right)+a\left(\phi_{\gamma_{1, w}}\left(n_{0}+n_{1}\right)-\phi_{\gamma_{1, w}}\left(n_{0}\right)\right) \\
= & \|x\|_{\gamma_{1, w}}=1 .
\end{aligned}
$$

Similarly, we may show that $\|z\|_{\gamma_{1, w}}=1$. Therefore, in view of assumption that $x$ is an extreme point of $B_{\gamma_{1, w}}$ we conclude a contradiction, which proves our claim. In case when $n_{0}>1$ we assume that $w(n)=0$ for all $n \in\left\{1, \ldots, n_{0}-1\right\}$. Then, for $a \in\left(0, x^{*}\left(n_{0}\right)\right)$ we define

$$
y=x^{*}+a \chi_{\{1\}}-a \chi_{\left\{n_{0}\right\}} \text { and } z=x^{*}-a \chi_{\{1\}}+a \chi_{\left\{n_{0}\right\}} \text {. }
$$

Next, it is clearly observe that $y \neq z, x=(y+z) / 2, y^{*}=y=z^{*}$ and

$$
\|z\|_{\gamma_{1, w}}=\|y\|_{\gamma_{1, w}}=\sum_{n=n_{0}}^{\infty} \frac{w(n)}{n} \sum_{j=1}^{n}\left(x^{*}(j)+a \chi_{\{1\}}(j)-a \chi_{\left\{n_{0}\right\}}(j)\right)=1 .
$$

Consequently, by assumption that $x$ is an extreme point of $B_{\gamma_{1, w}}$ we have a contradiction. So, this implies that if $n_{0}>1$ then it is needed $W\left(n_{0}-1\right)>0$.. Now, assume that $x \in \gamma_{1, w}$ and satisfies (7). For simplicity of our notation we denote $c=1 / \gamma_{1, w}\left(n_{0}\right)$. If $n_{0}=1$, then by Theorem 4.4 we conclude that $x$ is an extreme point of $B_{\gamma_{1, w}}$. Consider that $n_{0}>1$. Suppose that $y, z \in S_{\gamma_{1, w}}, y \neq z$ and $x=(y+z) / 2$. We claim that $y(i)=z(i)=0$ for all $i>n_{0}$. Indeed, if $y(i)>0$ for some $i>n_{0}$, then it is obvious that $z(i)=-y(i)<0$ for some $i>n_{0}$. Next, defining two elements

$$
u=y \chi\left\{i \in \mathbb{N}: i \leq n_{0}\right\} \quad \text { and } \quad v=z \chi\left\{i \in \mathbb{N}: i \leq n_{0}\right\}
$$

we have $x=(u+v) / 2$. On the other hand, by Theorem 4.4 we infer that $\|u\|_{\gamma_{1, w}}<$ $\|y\|_{\gamma_{1, w}}=1$ and $\|v\|_{\gamma_{1, w}}<\|z\|_{\gamma_{1, w}}=1$. In consequence, we get

$$
1=\|x\|_{\gamma_{1, w}}=\frac{1}{2}\|u+v\|_{\gamma_{1, w}} \leq \frac{\|u\|_{\gamma_{1, w}}+\|v\|_{\gamma_{1, w}}}{2}<1
$$


which yields a contradiction and proves our claim. Now, define

$$
\begin{aligned}
& I_{1}=\left\{i \in \mathbb{N}, i \leq n_{0} ; y(i)>c\right\}, \\
& I_{2}=\left\{i \in \mathbb{N}, i \leq n_{0} ; y(i)=c\right\}, \\
& I_{3}=\left\{i \in \mathbb{N}, i \leq n_{0} ; y(i)<c\right\} .
\end{aligned}
$$

We can easily notice that $y, z \in \gamma_{1, w}^{+}$. Indeed, if it is not true then we may define $u, v \in \gamma_{1, w}^{+}$such that $u \leq|y|, u \neq|y|$ and $v \leq|z|, v \neq|z|$ and also $x=(u+$ $v) / 2$. Therefore, by Theorem 4.4 we obtain a contradiction. Next, since $\gamma_{1, w}$ is strictly monotone and $y \in S_{\gamma_{1, w}}, y \neq x$ we observe that $\operatorname{card}\left(I_{1}\right)>0$ and $\operatorname{card}\left(I_{3}\right)>0$, whence $y(1)>y\left(n_{0}\right)$. Without loss of generality we may assume that $y=y^{*}$. Then, we have

$$
\begin{aligned}
1 & =\sum_{n=1}^{n_{0}-1} y^{* *}(n) w(n)+\sum_{i=1}^{n_{0}} y(i) \sum_{n=n_{0}}^{\infty} \frac{w(n)}{n} \\
& =\sum_{n=1}^{n_{0}-1} \sum_{i=1}^{n} y(i) \frac{w(n)}{n}+\sum_{i=1}^{n_{0}} y(i) \sum_{n=n_{0}}^{\infty} \frac{w(n)}{n} .
\end{aligned}
$$

Moreover, by assumption that $z \in S_{\gamma_{1, w}}$ and $x=(y+z) / 2$ it follows that $z(i)=$ $2 c-y(i)$ for any $i \in\left\{1, \ldots, n_{0}\right\}$ and $z(i)=0$ for all $i>n_{0}$. Thus, we obtain

$$
z^{*}(n)=\left(2 c-y\left(n_{0}+1-n\right)\right) \chi_{\left\{i \in \mathbb{N}: i \leq n_{0}\right\}}(n)
$$

for every $n \in \mathbb{N}$. Consequently, we have

$$
\begin{aligned}
1 & =\sum_{n=1}^{n_{0}} z^{* *}(n) w(n)+\sum_{i=1}^{n_{0}} z(i) \sum_{n=n_{0}+1}^{\infty} \frac{w(n)}{n} \\
& =\sum_{n=1}^{n_{0}}\left(2 c n-\sum_{i=1}^{n} y\left(n_{0}+1-i\right)\right) \frac{w(n)}{n}+\left(2 c n_{0}-\sum_{i=1}^{n_{0}} y(i)\right) \sum_{n=n_{0}+1}^{\infty} \frac{w(n)}{n} \\
& =2 c \phi_{\gamma_{1, w}}\left(n_{0}\right)-\sum_{n=1}^{n_{0}} \sum_{i=1}^{n} y\left(n_{0}+1-i\right) \frac{w(n)}{n}-\sum_{i=1}^{n_{0}} y(i) \sum_{n=n_{0}+1}^{\infty} \frac{w(n)}{n}
\end{aligned}
$$

Hence, by definition of $c$ we obtain that

$$
1=\sum_{n=1}^{n_{0}-1} \sum_{i=1}^{n} y\left(n_{0}+1-i\right) \frac{w(n)}{n}+\sum_{i=1}^{n_{0}} y(i) \sum_{n=n_{0}}^{\infty} \frac{w(n)}{n}
$$


Furthermore, since $y=y^{*}$ and $y(1)>y\left(n_{0}\right)$, we infer that for every $n<n_{0}$,

$$
\sum_{i=1}^{n} y(i)>\sum_{i=1}^{n} y\left(n_{0}+1-i\right)
$$

In consequence, since $W\left(n_{0}-1\right)>0$, by (9) and (10) we conclude

$$
\begin{aligned}
1 & =\sum_{n=1}^{n_{0}-1} \sum_{i=1}^{n} y(i) \frac{w(n)}{n}+\sum_{i=1}^{n_{0}} y(i) \sum_{n=n_{0}}^{\infty} \frac{w(n)}{n} \\
& >\sum_{n=1}^{n_{0}-1} \sum_{i=1}^{n} y\left(n_{0}+1-i\right) \frac{w(n)}{n}+\sum_{i=1}^{n_{0}} y(i) \sum_{n=n_{0}}^{\infty} \frac{w(n)}{n}=1,
\end{aligned}
$$

which gives us a contradiction and finishes the proof.

\section{Application}

This section is devoted to a relationship between the existence set and onecomplemented subspaces of the sequence Lorentz space $\gamma_{1, w}$. Next, we investigate complete criteria for the dual and predual spaces of the Lorentz space $\gamma_{1, w}$. Moreover, we present a complete characterization of smooth points in the sequence Lorentz space $\gamma_{1, w}$ and its dual space and predual space. Finally, we show full criteria for extreme points in the dual space of the sequence Lorentz space $\gamma_{1, w}$.

First, let us recall some basic definitions and notations that corresponds to the best approximation. Let $X$ be a Banach space and $C \subset X$ be a nonempty set. A continuous surjective mapping $P: X \rightarrow C$ is called a projection onto $C$, whenever $\left.P\right|_{C}=\mathrm{Id}$, i.e., $P^{2}=P$. Given a subspace $V$ of a Banach space $X$, by $P(X, V)$ we denote the set of all linear bounded projections from $X$ onto $V$. Let us recall that a closed subspace $V$ of a Banach space $X$ is said to be one-complemented if there exists a norm one projection $P \in P(X, V)$. A set $C \subset X$ is said to be an existence set of the best approximation if for any $x \in X$ we have

$$
R_{C}(x)=\left\{y \in C:\|x-y\|_{X}=\inf _{c \in C}\|x-c\|_{X}\right\} \neq \varnothing .
$$

It is obvious that any one-complemented subspace is an existence set. The converse in general is not true. By a deep result of Lindenstrauss [15] there exists a Banach space $X$ and a linear subspace $V$ of $X$ such that $V$ is an existence set in $X$ and $V$ is not one-complemented in $X$. However, if $X$ is a smooth Banach space both notions are equivalent see [1, Proposition 5]). It is worth noticing that one can find in the literature concerning one-complemented subspaces a survey paper [18]. We will show that both notions are equivalent in $\gamma_{1, w}$, which is obviously not a smooth space.

First, we establish an identity between the sequence Lorentz spaces $\gamma_{1, w}$ and $d_{1, v}$ for some nonnegative sequences $w$ and $v$. 
Remark 5.1 Assuming that $w=(w(n))_{n \in \mathbb{N}}$ is a nonnegative weight sequence, we may easily observe that the identity $I$ is a surjective isometry from the sequence Lorentz space $\gamma_{1, w}$ onto the sequence Lorentz space $d_{1, v}$, where $v=(v(n))_{n \in \mathbb{N}}$ is given by

$$
v(i)=\sum_{n=i}^{\infty} \frac{w(n)}{n} \text { for any } i \in \mathbb{N}
$$

Indeed, taking $x \in \gamma_{1, w}$ we observe that

$$
\|x\|_{\gamma_{1, w}}=\sum_{n=1}^{\infty} \frac{w(n)}{n} \sum_{i=1}^{n} x^{*}(i)=\sum_{i=1}^{\infty} x^{*}(i) \sum_{n=i}^{\infty} \frac{w(n)}{n}=\sum_{n=1}^{\infty} x^{*}(n) v(n)=\|x\|_{d_{1, v}} .
$$

On the other hand, assuming that $v=(v(n))_{n \in \mathbb{N}}$ is a decreasing sequence such that $\lim _{n \rightarrow \infty} v(n)=0$ we may define a sequence $w$ by

$$
w(n)=n(v(n)-v(n+1)) \text { for any } n \in \mathbb{N}
$$

and show analogously that the identity $I$ is a surjective isometry from $d_{1, v}$ onto $\gamma_{1, w}$.

Theorem 5.2 Let $w$ be a nonnegative weight sequence and let $V \subset \gamma_{1, w}, V \neq\{0\}$ be a linear subspace. If $V$ is an existence set, then $V$ is one-complemented.

Proof Let $v$ be a nonnegative sequence given by (11). Then, by Remark 5.1 we get that the identity $I$ is a surjective isometry between $\gamma_{1, w}$ and $d_{1, v}$. Hence, since $V$ is an existence set in $\gamma_{1, w}$, by [10, Lemma 3.4] it follows that $V \neq\{0\}$ is an existence set in $d_{1, v}$. In consequence, by [10, Theorem 3.10] we infer that $V$ is one-complemented in $d_{1, v}$. Finally, applying again [10, Lemma 3.4] we get that $V$ is one-complemented in $\gamma_{1, w}$.

Now, we present the necessary and sufficient condition for the dual space of the Lorentz space $\gamma_{1, w}$ and the isometric isomorphism between the Marcinkiewicz space and the dual space of $\gamma_{1, w}$. It is worth mentioning that in case of the Lorentz space $d_{1, w}$ the similar result (see [14, Theorem 5.2]) was established under assumption that $d_{1, w}$ is separable.

Theorem 5.3 Let $w=(w(n))_{n \in \mathbb{N}}$ be a nonnegative weight sequence and let $\phi_{\gamma_{1, w}}$ be the fundamental sequence of the sequence Lorentz space $\gamma_{1, w}$. Then $W(\infty)=\infty$ if and only if every linear bounded functional $f$ on $\gamma_{1, w}$ has the form

$$
f(x)=\sum_{n=1}^{\infty} x(n) y(n) \quad \text { for any } \quad x \in \gamma_{1, w}, \quad \text { and } \quad\|f\|_{\gamma_{1, w}^{*}}=\|y\|_{m_{\psi}},
$$

where $y \in m_{\psi}$ and $\psi(n)=n / \phi_{\gamma_{1, w}}(n)$ for every $n \in \mathbb{N}$. 
Proof Sufficiency Suppose that $W(\infty)<\infty$. We claim that $\ell^{\infty} \hookrightarrow \gamma_{1, w}$. Indeed, taking $x=\chi_{\mathbb{N}}$ it is easy to see that $x^{* *}=x$ and $\|x\|_{\gamma_{1, w}}=W(\infty)<\infty$, which implies our claim. Let $f \in \gamma_{1, w}^{*}$. Then, by assumption there exists $y \in m_{\psi}$ such that

$$
\|f\|_{\gamma_{1, w}^{*}}=\|y\|_{m_{\psi}} \geq \frac{1}{\phi_{\gamma_{1, w}}(n)} \sum_{i=1}^{n} y^{*}(k)
$$

for all $n \in \mathbb{N}$. Next, in view of the inequality

$$
W(n) \leq \phi_{\gamma_{1, w}}(n) \leq W(\infty)<\infty
$$

for every $n \in \mathbb{N}$, it follows that $\phi_{\gamma_{1, w}}(\infty)=W(\infty)<\infty$. Thus , we have

$$
\phi_{\gamma_{1, w}}(\infty)\|f\|_{\gamma_{1, w}^{*}} \geq \sum_{i=1}^{\infty} y^{*}(k),
$$

whence $y \in \ell^{1}$. Therefore, we observe that $m_{\psi} \hookrightarrow \ell^{1}$. Moreover, since $\gamma_{1, w}$ and $m_{\psi}$ are symmetric by [2, Corollary 6.8] we conclude that $\gamma_{1, w} \hookrightarrow \ell^{\infty}$ and $\ell^{1} \hookrightarrow m_{\psi}$. Hence, since $\ell^{\infty}$ is the dual space of $\ell^{1}$ (see [16]) we have a contradiction.

Necessity Since $W(\infty)=\infty$, by Theorem 4.1 it follows that $\gamma_{1, w}$ is order continuous. Next, in view of Remark 5.1 the identity is a surjective isometry between $\gamma_{1, w}$ and $d_{1, v}$ where $v$ is given by (11). Finally, by [14, Theorem 5.2] we finish the proof.

We research a complete characterization of the predual space of the Lorentz space $\gamma_{1, w}$. It is worth noticing that the first part of the proof of the next theorem is an immediate consequence of [9, Theorem 3.4], in which authors have proved that the Marcinkiewicz space $m_{\psi}$ is the bidual space of $m_{\psi}^{0}$ if and only if $\psi(\infty)=\infty$. Additionally, we answer the essential question under which condition does the isometric isomorphism exist between the Lorentz space $\gamma_{1, w}$ and the dual space of the Marcinkiewicz space $m_{\psi}^{0}$.

Theorem 5.4 Let $w$ be a nonnegative weight sequence. The Marcinkiewicz space $m_{\psi}^{0}$ is the predual of the sequence Lorentz space $\gamma_{1, w}$ if and only if $W(\infty)=\infty$, where

$$
\psi(n)=\frac{n}{\phi_{\gamma_{1, w}}(n)} \text { for any } n \in \mathbb{N}
$$

Additionally, if $W(\infty)=\infty$ then there exists an isometry between the sequence Lorentz space $\gamma_{1, w}$ and the dual space $\left(m_{\psi}^{0}\right)^{*}$ of the Marcinkiewicz space $m_{\psi}^{0}$.

Proof First, we define for any $i \in \mathbb{N}$,

$$
v(i)=\sum_{k=i}^{\infty} \frac{w(k)}{k} \text {. }
$$


Clearly, $(v(i))_{i \in \mathbb{N}}$ is a decreasing sequence and $0 \leq v(i)<\infty$ for all $i \in \mathbb{N}$. Hence, by Remark 5.1 we obtain that

$$
\phi_{\gamma_{1, w}}(n)=\left\|\chi_{[1, n]}\right\|_{\gamma_{1, w}}=\left\|\chi_{[1, n]}\right\|_{d_{1, v}}=\sum_{i=1}^{n} v(i)
$$

for all $n \in \mathbb{N}$. Next, since $\psi(n)=n / \phi_{\gamma_{1, w}}(n)$ for each $n \in \mathbb{N}$, we have

$$
\begin{aligned}
m_{\psi} & =\left\{x=(x(n))_{n \in \mathbb{N}}: \sup _{n \in \mathbb{N}}\left\{x^{* *}(n) \psi(n)\right\}<\infty\right\} \\
& =\left\{x=(x(n))_{n \in \mathbb{N}}: \sup _{n \in \mathbb{N}}\left\{\frac{\sum_{i=1}^{n} x^{*}(i)}{\phi_{\gamma_{1, w}}(n)}\right\}<\infty\right\} .
\end{aligned}
$$

Thus, by [9, Theorem 3.4] it follows that $m_{\psi}$ is the bidual of $m_{\psi}^{0}$ if and only if $\phi_{\gamma_{1, w}}(\infty)=\infty$. Now, we claim that $W(\infty)=\infty$ if and only if $\phi_{\gamma_{1, w}}(\infty)=\infty$. Indeed, it is easy to see that for any $n \in \mathbb{N}$,

$$
W(n) \leq \phi_{\gamma_{1, w}}(n) \leq \sum_{i=1}^{n} w(i)+n \sum_{i=n+1}^{\infty} \frac{w(i)}{n}=W(\infty)
$$

which implies our claim. Therefore, according to Theorem 5.3 we obtain that the Marcinkiewicz space $m_{\psi}^{0}$ is the predual of the sequence Lorentz space $\gamma_{1, w}$ if and only if $W(\infty)=\infty$. Now, we show that there exists an isometry between $\left(m_{\psi}^{0}\right)^{*}$ and $\gamma_{1, w}$. First, since $\phi_{\gamma_{1, w}}(\infty)=\infty$, in view of [9, Theorem 3.2] it follows that $m_{\psi}^{0}$ is a nontrivial subspace of all order continuous elements of $m_{\psi}$. Then, defining for any $x \in \gamma_{1, w}$ the linear mapping $f_{x}$ by

$$
f_{x}(y)=\sum_{n=1}^{\infty} x(n) y(n) \text { for any } y \in m_{\psi}^{0},
$$

and proceeding analogously as in Theorem 5.3 we are able to show that

$$
\left|f_{x}(y)\right| \leq\|y\|_{m_{\psi}}\|x\|_{\gamma_{1, w}}
$$

for any $y \in m_{\psi}^{0}$. On the other hand, it is well known that there exists $\sigma: \mathbb{N} \rightarrow \mathbb{N}$ a permutation such that $x^{*}(n)=|x \circ \sigma(n)|$ for all $n \in \mathbb{N}$. Define

$$
y(n)= \begin{cases}\operatorname{sgn}(x(n)) v\left(\sigma^{-1}(n)\right) & \text { if } n \in \sigma(\mathbb{N}), \\ 0 & \text { otherwise }\end{cases}
$$


for any $n \in \mathbb{N}$. Then, we have

$$
f_{x}(y)=\sum_{n=1}^{\infty} x(n) y(n)=\sum_{n \in \sigma(N)}|x(n)| v \circ \sigma^{-1}(n)=\sum_{n=1}^{\infty} x^{*}(n) v(n)=\|x\|_{\gamma_{1, w}},
$$

whence, according to (12) we finish the proof.

We investigate a full criteria for smooth points in the sequence Lorentz space $\gamma_{1, w}$ and its dual and predual spaces. First, let us notice that by [10, Theorem 1.10] and by Remark 5.1, the next theorem follows immediately.

Theorem 5.5 Let $w$ be a nonnegative weight sequence and let $x \in S_{\gamma_{1, w}}$. Then, an element $x$ is a smooth point in $\gamma_{1, w}$ if and only if the following conditions are satisfied

(i) $\operatorname{card}(\operatorname{supp}(x))=\infty$.

(ii) If there is $n \in \mathbb{N}$ such that $w(n)>0$, then $x^{*}(n)>x^{*}(n+1)$.

Theorem 5.6 Let $w$ be a nonnegative weight sequence and $\psi(n)=n / \phi_{\gamma_{1, w}}(n)$ for any $n \in \mathbb{N}$ and $x \in S_{m_{\psi}^{0}}$. Then, an element $x$ is a smooth point in $m_{\psi}^{0}$ if and only if

$$
\operatorname{card}\left\{n \in \mathbb{N}: x^{* *}(n) \psi(n)=1\right\}=1 .
$$

Proof Let $v$ be a sequence given by (11) and let $V(n)=\sum_{i=1}^{n} v(i)$. Then, by Remark 5.1 we easily observe that $V(n)=\phi_{\gamma_{1, w}}(n)=\frac{n}{\psi(n)}$ for every $n \in \mathbb{N}$ and

$$
m_{\psi}^{0}=\left\{x \in m_{\psi}: \lim _{n \rightarrow \infty} \frac{1}{V(n)} \sum_{i=1}^{n} x^{*}(i)=0\right\} .
$$

Hence, in view of [10, Theorem 1.5] we complete the proof.

Directly, by [10, Theorem 1.9] and Remark 5.1 and also Theorem 5.3 we infer the following theorem.

Theorem 5.7 Let $w$ be a nonnegative weight sequence and $\psi(n)=n / \phi_{\gamma_{1, w}}(n)$ for any $n \in \mathbb{N}$ and $x \in S_{\gamma_{1, w}^{*}}$. Then, an element $x$ is a smooth point in $B_{\gamma_{1, w}^{*}}$ if and only if there exists $n_{0} \in \mathbb{N}$ such that

$$
x^{* *}\left(n_{0}\right) \psi\left(n_{0}\right)=1>\sup _{n \neq n_{0}}\left\{x^{* *}(n) \psi(n)\right\} .
$$

The last essential application of Theorem 5.3 and Remark 5.1, in view of [10, Theorem 2.2], is the next result which presents an equivalent condition for extreme points in the dual space $\gamma_{1, w}^{*}$ of the sequence Lorentz space $\gamma_{1, w}$.

Theorem 5.8 Let $w$ be a nonnegative weight sequence. Then, $x \in S_{\gamma_{1, w}^{*}}$ is an extreme point of $B_{\gamma_{1, w}^{*}}$ if and only if $x^{*}(n)=\sum_{i=n}^{\infty} \frac{w(i)}{i}$ for all $n \in \mathbb{N}$. 
Remark 5.9 Although applying [10, Theorem 2.6] and Remark 5.1 we are able to find successfully an equivalent condition for an extreme point in the sequence Lorentz space $\gamma_{1, w}$, with $w$ a nonnegative weight sequence, we present the proof of this problem with all details (see Theorem 4.7). It is worth mentioning that the techniques, that were presented in the proof of Theorem 4.7, might be interesting for readers and applicable to search a complete characteristic of an extreme point in $\gamma_{p, w}$ with $1<p<\infty$.

Acknowledgements Maciej Ciesielski is supported by the Ministry of Science and Higher Education of Poland, Grant Number 04/43/DSPB/0094.

Open Access This article is distributed under the terms of the Creative Commons Attribution 4.0 International License (http://creativecommons.org/licenses/by/4.0/), which permits unrestricted use, distribution, and reproduction in any medium, provided you give appropriate credit to the original author(s) and the source, provide a link to the Creative Commons license, and indicate if changes were made.

\section{References}

1. Beauzamy, B., Maurey, B.: Points minimaux et ensembles optimaux dans les espaces de Banach. J. Funct. Anal. 24(2), 107-139 (1977)

2. Bennett, C., Sharpley, R.: Interpolation of Operators, Pure and Applied Mathematics, vol. 129. Academic, Boston (1988)

3. Ciesielski, M.: On geometric structure of symmetric spaces. J. Math. Anal. Appl. 430(1), 98-125 (2015)

4. Ciesielski, M.: Relationships between $K$-monotonicity and rotundity properties with application. J. Math. Anal. Appl. (2018). https://doi.org/10.1016/j.jmaa.2018.05.008

5. Ciesielski, M., Kamińska, A., Kolwicz, P., Płuciennik, R.: Monotonicity and rotundity of Lorentz spaces $\Gamma_{p, w}$. Nonlinear Anal. 75(5), 2713-2723 (2012)

6. Ciesielski, M., Kamińska, A., Płuciennik, R.: Gâteaux derivatives and their applications to approximation in Lorentz spaces $\Gamma_{p, w}$. Math. Nachr. 282(9), 1242-1264 (2009)

7. Davis, W.J., Enflo, P.: Contractive Projections on $l_{p}$ Spaces, Analysis at Urbana (Urbana, IL, 19861987), London Mathematical Society Lecture Note Series 137, vol. I, pp. 151-161. Cambridge University Press, Cambridge (1989)

8. Jamison, J.E., Kamińska, A., Lewicki, G.: One-complemented subspaces of Musielak-Orlicz sequence spaces. J. Approx. Theory 130(1), 1-37 (2004)

9. Kamińska, A., Lee, H.J.: M-Ideal Properties in Marcinkiewicz Spaces, Comment. Math. (Prace Mat.) Tomus specialis in Honorem Juliani Musielak, pp. 123-144 (2004)

10. Kamińska, A., Lee, H.J., Lewicki, G.: Extreme and smooth points in Lorentz and Marcinkiewicz spaces with applications to contractive projections. Rocky Mt. J. Math. 39(5), 1533-1572 (2009)

11. Kamińska, A., Lewicki, G.: Contractive and optimal sets in modular spaces. Math. Nachr. 268, 74-95 (2004)

12. Kamińska, A., Maligranda, L.: On Lorentz spaces $\Gamma_{p, w}$. Isr. J. Math. 140, 285-318 (2004)

13. Kamińska, A., Maligranda, L.: Order convexity and concavity of Lorentz spaces $\Lambda_{p, w}, 0<p<\infty$. Stud. Math. 160(3), 267-286 (2004)

14. Krein, S.G., Petunin, Yu.I., Semenov, E.M.: Interpolation of Linear Operators. Translated from the Russian by J. Szúcs. Translations of Mathematical Monographs, vol. 54. American Mathematical Society, Providence (1982)

15. Lindenstrauss, J.: On projections with norm 1-an example. Proc. Am. Math. Soc. 15, $403-406$ (1964)

16. Lindenstrauss, J., Tzafriri, L.: Classical Banach Spaces. II. Function Spaces. Ergebnisse der Mathematik und ihrer Grenzgebiete [Results in Mathematics and Related Areas], vol. 97. Springer, Berlin (1979)

17. Lorentz, G.G.: On the theory of spaces $\Lambda$. Pac. J. Math. 1, 411-429 (1951)

18. Randrianantoanina, B.: Norm-one projections in Banach spaces. International conference on mathematical analysis and its applications (Kaohsiung, 2000). Taiwan. J. Math. 5(1), 35-95 (2001)

19. Royden, H.L.: Real Analysis, 3rd edn. Macmillan Publishing Company, New York (1988) 
20. Sparr, A.: On the Conjugate Space of the Lorentz Space $L(\phi, q)$, (English Summary) Interpolation Theory and Applications, Contemporary Mathematics, vol. 445, pp. 313-336. American Mathematical Society, Providence (2007) 